\title{
The Invisibility of Race: A Pragmatic Approximation to the Concept
}

\author{
Liza Hayes \\ Department of Sociology, University of Miami, United States
}

Copyright $\bigcirc 2017$ by authors, all rights reserved. Authors agree that this article remains permanently open access under the terms of the Creative Commons Attribution License 4.0 International License

\begin{abstract}
Our society is racially characterized by subtle forms of discrimination and prejudice. At the present time, people have adopted an attitude of racial denial, reinforcing an attitude of racial invisibility. This study is the first qualitative attempt to measure Franklin's paradigm, the invisible paradigm. Using data from the African- American study $(\mathrm{N}=2,864)$, I examine the relation between race invisibility with discrimination, social recognition, and group affiliation. Three hypotheses were tested: (1) discrimination affects the individual's perception of race visibility, micro-aggression, and group affiliation; (2) social recognition is related to group affiliation and visibility; and (3) group affiliation mediates the relationship between discrimination and visibility. Results from the path analysis show that perceived discrimination has a negative and significant association with micro-aggression and visibility and a positive and significant association with group affiliation. Group affiliation mediated the relationship between discrimination and visibility. Social recognition, however, does not affect race visibility directly. Its effect is mediated through group affiliation. This study contributes to the social knowledge of the way discrimination affects minority ethnic groups' micro-aggression, group affiliation, and racial visibility.
\end{abstract}

Keywords Race, Discrimination, Micro-aggression

\section{Introduction}

For most people, racism is perceived as a thing of the past and the idea of a racialized society an unacceptable tough, as the old fashioned type of racism seems to have vanished [1]. However, as the sociologist Eduardo Bonilla-Silva [1] explains, racism is still a problem in today's society; a new form has emerged, morphed into more insidious expressions of racial discrimination that take place at the individual level, in face-to-face interactions: now you see black people, now you don't see them [1]. As a result, minorities are becoming more and more invisible $[2,3,4,5]$. The invisibility, as the great sociologist W.E.B. DuBois [6] and the novelist Ralph Ellison [7] pointed out many decades ago, is not a simple a matter of being overlook, it is, paradoxically, a consequence of conspicuousness. The implications of invisibility are evident in today's society, as John L. Seigenthaler explains at the Fulbright Enrichment Seminar in Nashville, TN, 2012:

"I buy the bus ticket just as this black woman who was carrying a tub. She might be carrying clothes to iron, so the white children could wear [them] to school the next day. She might also be carrying food for her children to have dinner, food given by her white master. There she was, trying to find a way into the back of the bus. One might not believe me, but, I did not see her. No, I did not see her. She was invisible to me, just as [if] she was not there. I was blind then. And today, I can reflect back and say that we were in a country based on a corrupted society's core; in a corrupted culture."

The concept of invisibility was developed by the psychologist Anderson J. Franklin [2], the Invisible Syndrome. According to this theory, invisibility occurs when someone, particularly a black person, is disregarded because of others' prejudice and discrimination and the perpetrators' presumptions about Blacks are filled with all types of social and personal stereotypes, leaving the real person invisible. As Franklin [2] explains, the process of making invisible the other person is produced by subtle and insidious behaviors, not always recognized by the perpetrator, referred to as micro-aggression [8]. Micro-aggression are a modern and covert form of discrimination that increase minorities' sense of invisibility.

Another manifestation of racism, as McDonald [5] defines it, is conspicuous visibility, or hypervisibility. Hypervisibility occurs when racial/ethnic minorities are exposed as unique "others" [5]. The authors argue that minorities' experiences as targets of racism fused both invisibility and hypervisibility to form what they defined as invisibility [5]. Although the racism associated with visibility is not clear, for it involves individual behavior as it 
occurs at the personal level in face-to-face interactions, it is as damaging as the "old" institutional racism because it is a reminder that social and cultural divisions still exist in today's society $[2,4]$.

This study seeks to highlight the effect invisibility experiences have on minorities, using Franklin's [2] syndrome of invisibility paradigm. The purpose of this study is to determine to what extent minorities perceived being (in) visible and experienced discrimination in their everyday lives.

\section{(In)Visibility of Race}

The Civil Rights Act of 1964 gave society the basis to combat discrimination through tailoring and implementing anti-discriminatory policies. As the old, overt type of discrimination can result in legal penalties, in today's society, new covert, subtle, and complex types of mechanisms to perpetuate the racial/ethnic differentiation have developed [1, 3]. Bonilla-Silva [1] believes that after the civil rights era, racial inequality is presented as colorblindness racism. Color-blindness is presented as a non-discriminatory behavior, but the discourse is not such. On the contrary, color-blind racism incorporates a racial/ethnic denial manifested in discursive strategies that serve to maintain white racial hegemony [1]. The argument of this racism is that whites do not see color, claiming that neither race nor racism are relevant in today's society [1].

Because blatant racism has been on the decline since the Jim Crow era, the color-blind form of racism becomes hard to recognize and thus harder to combat. Scholars argue that even though overt racism has diminished, everyday racism has increased through individual practices $[1,2,5]$. Bonilla-Silva [2] calls this color-blindness a form of racism that functions without racists but one that protects the status quo and maintains the current racial hierarchy. Color-blindness masks today's racial inequality with the rhetoric of equal opportunity and fair treatment [2]. Color-blind racism, according to Bonilla-Silva [2], is more detrimental than the old overt form of racism because the people engaged in it are unaware of it, and thus the harm it causes has lasting effects on racial/ethnic minorities, as it perpetuates racial/ethnic stereotypes and further contributes to $\mathrm{racial} / \mathrm{ethnic}$ inequalities.

Though not often intentional in nature, this new form of discrimination has become pervasive in the lives of minorities, for it negates the existence of the person $[1,3]$. As Mills [9] clearly explains it, in a post-racism era the dominant group determines when a minority presence becomes absence, or when it is seen as unseen. As Mills [9] noted, most majorities do see minorities, but they can live as though these minorities are invisible. This new type of racism has resulted in significant negative consequences for ethnic/racial minorities, particularly for blacks, causing social distancing, which often leads to the experience of marginalization $[2,5]$, where ethnic/racial minorities come to the realization that they are not being recognized because of prejudice and racism; therefore they are invisible [2].

Anderson Franklin [2] has elaborated a grounded-theoretical model of racial/ethnic invisibility. The author claims that as a result of individual encounters with intentional or unintentional discriminatory behavior, minorities become invisible $[2,3]$. The experience of social invisibility is mostly cited as a product of social exclusion [5]. However, as it is well described in the most famous and most cited work of Ralph Ellison [7], Invisible Man, invisibility encompasses multiple spheres of minorities' lives, where any traces of ethnic/racial identity are not totally suppressed and must therefore remain invisible[5]. Today's social belief is that this society is post-racism, but there is still racism, a racism that challenges policies and is at the individual level, [1] The deleterious consequences of this new form of discriminatory behavior are immeasurable $[5,10,11,12]$, as invisibility, then turned into a sentiment, leads to significant psychological consequences for minorities [5], because these minorities struggle with the feelings of not being worthy of respect, causing confusion, self-doubt, and self-denigration, even as they continue to demand recognition $[2,4,10,11$, 14].

In the struggle for recognition, Franklin [2] believes two aspects of black people's lives are both challenged and reinforced: ethnic belonging and social recognition. Racial/ethnic belonging is defined as the shared common cultural heritage a person has with a particular group $[2,3]$. Invisibility either amplifies or minimizes minorities' feelings of belonging. As minorities encounter invisibility behavior, two approaches are taken: strengthen the ethnic/racial identity or slowly assimilate into the dominant culture. The first, racial/ethnic belonging, describes the process of moving from a negative emphasis on individual racial identity to a more highly emphasized collective and positive perspective.

Group affiliation is a psychological strategy used by minorities to reinforce their ethnicity as a mechanism to counter the negative impact of invisibility [2, 3, 13]. On the one hand, members of the group become more and more aware of the particularities proper to their ethnic/racial and cultural background, which is reinforced by other members of the group. This process mitigates the negative impact of the invisibility. The end result of the social belonging is ethnic legitimacy, which employs social distancing from the oppressor [2, 3]. Through the use of rationalized interpretations of social behavior, minorities challenge the social ontology and develop a new behavior, such as getting excited, talking out loud, and gesticulating [6]. Minorities that have experienced being either invisible or visible, according to Franklin [2, 3], create new social rules that suit themselves and provide them with a sense of belonging and ethnic legitimacy. On the other hand, as minorities become more aware of the social exclusion due to racism and prejudice, they work to assimilate, and as they become part of the dominant society, the particularities of their 
racial/ethnic and cultural heritage are removed, leading to their adopting the culture of the dominant group, becoming therefore visible [4]. However, the psychological effects of the feeling of displacement and isolation that result from being invisible persist, as ethnic/racial minorities fit imperfectly into the dominant culture into which they work to assimilate [5]. At the end, all that is left is a person stripped of anything objectionable to the dominant culture [10].

Another manifestation of discrimination is conspicuous visibility or hypervisibility, where ethnic/racial minorities are exposed as unique others [5]. This hypervisibility, explains Franklin [2], emanates from the naïveté of the dominant group and the lack of knowledge about the lives of minorities.

Invisibility and hypervisibility concepts are intertwined [5]. This association is embodied in the contradictory association between the invisible and hyper-visible dualism defined as (in)visibility [5]. This contradictory approach to racial/ethnic relations reflects the transition to a post-civil rights era; a period marked by the hypervisibility and invisibility of minorities. Paradoxically, while features of African-American culture become hyper-visible as a symbol of a post-civil rights era, these features are also invisible as they are not culturally relevant $[10,11]$.

It is the duality of visible or invisible that serves in this study to understand the dynamic relationship between discrimination and individual experiences of visibility or invisibility.

The second factor of invisibility is social recognition, which takes place within interpersonal interaction. It is in interpersonal interaction that people seek for validation and recognition, particularly for ethnic/racial minorities largely invisible. Although ethnic belonging offers a first scenario from which to be socially accepted, the greater society as a whole plays a more fundamental role [2, 3, 13]. Consequently, it is through the interaction with other members of the society that ethnic/racial minorities seek for social recognition. Positive social recognition occurs in social conjunctures or places and circumstances that provide a greater level of gratification and acceptance of the particularities of a person's racial/ethnic culture, increasing therefore the perception of visibility [3]. However, racial/ethnic minorities are subject in their everyday life to differential levels of social recognition due to prerogative perceptions [5] that exclude them from being fully members of society [9]. This experience has led to tension between ethnic/racial minorities and majorities, which has led to interracial verbal and nonverbal behavior that serves to reinforce the negative stereotype racial/ethnic minorities, have, and consequently leads to racial micro-aggression.

Racial micro-aggression are subtle acts or attitudes, which are often unintentional, that convey rudeness, insensitivity and demean a person's racial heritage or identity [8]. Sue and colleagues [8] describe micro-aggression as, "brief and commonplace daily verbal, behavioral, or environmental indignities, whether intentional or unintentional, that communicate hostile, derogatory, or negative racial slights and insults toward people of other race." The authors believe that micro-aggressions occur in a society that disregards a person's racial/ethnic culture and heritage and communicates, thus hostilely, derogatory racial/ethnic offenses [8]. Micro-aggressions appear in three forms: (1) microassault or explicit derogative and verbal/nonverbal insults to purposely hurt the recipient; (2) microinsults which are typically communicative messages that convey rudeness and insensitivities towards the recipient's racial/ethnic heritage or culture; and (3) micro-invalidations, which are negations of the thoughts, feelings, or the reality of the recipient[8]. A typical example of this type of micro-aggression is color-blind racism [2].

Contrary to contemporary society's beliefs, the color-blind statement "I don't see color" is, according to Bonilla-Silva [2], a clear manifestation of covert racism. This statement is emblematic of a racial ideology the author labeled as colorblind racism [2]. The author notes that whites avoid direct racial language to express racial views, or employ semantic moves to maneuver around conversation about race. Compared to the Jim Crow racism of earlier epochs, instead of calling ethnic/racial minorities by derogative names, color-blind racism otherizes racial/ethnic minorities [5] by using statements such as "these people are human, too" [2] or at the other extreme by ignoring racial/ethnic minorities as a mechanism to preserve and reproduce the social hierarchy and with it the system of white privilege [2].As a matter of fact, according to Bonilla-Silva[2], we are living in an era of color-blind racism, which serves as the ideological armor for a covert system of discrimination in today's society, the post-Civil Rights era [2]. Racism in this era is not a free-floating ideology, but rather a set of concepts strongly anchored in the social structure, which aims to maintain whites' privileges [2]. Racism and discrimination, therefore, are fundamental elements of the social dynamic, modifying the beliefs, attitudes, and behaviors of the majority toward racial/ethnic minorities.

One may postulate that whites have also adopted a new strategy, (in)visibility, which ignores the presence of racial/ethnic minorities. According to Bonilla-Silva [1], the lack of visibility of race is a frame that majorities use to explain and justify racially motivated behavior, or as Mills [9] defines it, as a mechanism to perpetuate power and to preserve the racial status quo and with it the racial hegemony of the majority, which perpetuates the invisibility of Afro-descendants.

Perpetrators of color-blind racism are often unaware of underlying racial bias due to their own social conditioning [2]. Due to such conditioning, many whites unknowingly perpetrate micro-aggressive acts without acknowledging the underlying racial bias [2]. Perpetrators of such racial micro-aggression sincerely believe that they are not being prejudiced. 
Racial/ethnic invisibility is not a social phenomenon, per se, but rather an individual response to multiple social processes. It is the act of recognizing (making visible) or denying (making invisible) "others" [5]. On the one hand, visibility empowers racial/ethnic minorities, when they are socially recognized. On the other hand, invisibility can result in an instrument of control and the perpetuation of the whites' racial status quo [2], when racial/ethnic minorities are not socially recognized.

The lack of social recognition is a type of micro-aggression. Social recognition depends on the dominant vision. For instance, a member of an ethnic/racial minority group that is not socially recognized, or is not seen in social encounters, is rendered socially invisible. This type of discrimination is the most powerful discriminatory act $[2$, $3,4]$, as the perpetrator has the power to recognize or to negate the existence of the other [9]. Social recognition, therefore, is the power to socially and individually determine if minorities are fully recognized as persons in the eyes of the dominant group [9]. This power, according to Orfali [13], is the major unconscious motive underlying human behavior and interaction. This type of aggression on the surface appears to be impersonal [5]; however, for minorities the cumulative nature of these seemingly insignificant behaviors can have deleterious effects on the self and create feelings of marginalization $[2,3]$.

\section{Materials and Methods}

This study on visibility of race takes into account the effects of perceived discrimination, racial micro-aggression, and group affiliation. A conceptual model hypothesizing the interrelationship between these variables is presented in Figure 1.

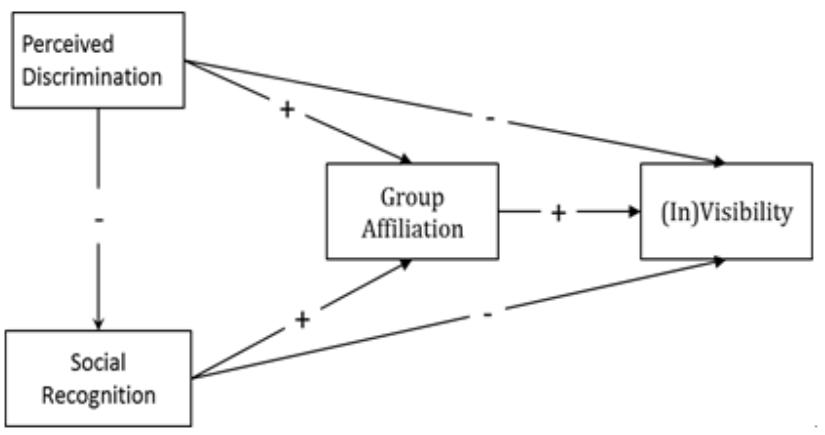

Figure 1. The Conceptual Path Analysis

Figure 1 represents the conceptual model of this study. In the diagram one-way arrows indicate a causal relationship from the determining variable to each variable dependent on it. Perceived discrimination and social recognition are exogenous variables. Their variance is assumed to be caused entirely by variables not defined in the causal model. The line connecting them indicates a relationship between these two variables that is causal, perceived discrimination causing micro-aggression, which can be due to sharing common racial/ethnic perceptions and beliefs.

Racial visibility and group affiliation ideology are endogenous variables in this model and their variance is considered to be explained in part by perceived discrimination and racial micro-aggression. Paths drawn to endogenous variables are directional (arrowhead on one end only). Variance in racial visibility is theorized to result from variations in perceived discrimination, social recognition, and group affiliation. Variation in group affiliation is theorized to be caused by variations in perceived discrimination and social recognition.

The main assumption of this study is that discrimination influences directly and indirectly black men's experience of invisibility through group affiliation, and that lack of social recognition affects visibility negatively. Perceived discrimination may decrease visibility directly and indirectly through social recognition and group affiliation. For instance, when minorities perceive discrimination, they become more susceptible to feeling invisible. Social recognition has a direct and negative association with visibility and an indirect association through group affiliation, which mediates the relationship. Similarly, racial/ethnic minorities that experience less social recognition are more susceptible to developing a sense of invisibility. The other alternative hypothesis proposed in the model (see Figure 1) is that group affiliation increases, visibility increases and, in all paths, group affiliation constitutes a protective response from the negative effects of discrimination and poor social recognition $[2,3,4,5]$.

This study uses data from the African American Men Survey [15]. A total of 2,864 adults across the U. S. participated in the telephone interviews conducted during March and April of 2006 by International Communications Research, in collaboration with The Washington Post, Kaiser Family Foundation, and Harvard University. Out of the total participants, 1,328 were black men, 507 black women, and 1,029 participants from other racial groups.

The exogenous variables are perceived discrimination and social recognition (see Figure 1). Perceived discrimination was measured using two questions: "Are you worried about being a victim of racial discrimination?" and "Is racial discrimination a big problem, a small problem or not a problem for black men today?" Responses ranged from $1=$ "very worried" to 4 = "not at all worried." Social recognition was measured using the question: "In your day-to-day life, how often do any of the following things happen to you because of your racial background? (a) You receive poorer service than other people at restaurants or stores and (b) People act as if they think you are not smart." Responses ranged from $0=$ "never" to $3=$ "very often." Responses were reversed so that high scores indicate higher levels of perceived discrimination and higher levels of social recognition.

The main endogenous variable of interest is racial (in)visibility, defined by the question "Considering 
everything, do you think it is a good time or a bad time to be a black man in America?" Responses were recoded such that 1 = "bad time" and $2=$ "good time." As responses increase, the sentiment of visibility increases and as responses decrease, the sentiment of invisibility increases. Group affiliation indicates the meaning racial/ethnic groups have for people. The following question was used to assess the centrality of group affiliation: "How about standing up for your racial or ethnic group?" This item was scored on a four Likert scale, with end points of $1=$ "very important" to $4=$ "not important at all." Responses were reversed. Finally, the key exogenous variables are gender $(1=$ male, $0=$ female $)$ and race $(1=$ blacks, $0=$ other race $)$.

\section{Data Analysis}

Path analysis is proposed as data strategy. A path analysis, which is a special case of Structural Equation Modeling, consists of a hierarchical multiple regression analysis [16]. For each endogenous variable a multiple regression model is conducted predicting visibility from all other variables which are hypothesized to have direct effects on visibility. Variables that are hypothesized to affect visibility directly and indirectly were not included in the analysis. The beta coefficients that resulted from these multiple regressions are the path coefficients shown in Figure 2. These coefficients are the numerical values of the path coefficients.

The data were analyzed by using LISREL 8.8 software. In addition to descriptive analysis, several statistical tests were used to evaluate different demographic, dependent and independent variables of the study, including mean age, and
Pearson correlation matrix. Also, path analysis of relationships, direct, indirect and total effects as well as fitness indexes were calculated. LISREL provides for all parameters the maximum-likelihood estimation that is the minimum difference between the observed covariance and the model estimate covariance $[16,17]$. LISREL 8.8 uses several fit indexes to assess how well the proposed path model fits the sample data: the Goodness of Fit Index (GFI), the Non-Normed Fit Index (NNFI), the Comparative Fit Index (CFI), and the Adjust Goodness-of-it Index (AGFI). This index is very sensitive to model misspecification (Xitao, Thompson, and Wang, 1999). Values above .90 indicate an optimal fit [18, 19]. The Root Mean Square of Approximation (RMSEA), which is a standardized summary of the average covariance residuals and takes into account the error of approximation in the population, is used to measure the fit of the model. As the average discrepancy between the observed and predicted covariance increases, so does the value of the RMSEA. When the fit of the model is perfect, the RMSEA equals zero. Values less than .05 indicate good fit, and values of .08 or less, indicate reasonable errors of approximation in the population; whereas, values of .08 to .10 indicate mediocre fit, and values above .10 indicate a poor fit $[18,19,20]$.

\section{Results}

Descriptive statistics for the total sample correlation among variables are shown in Table 1.

Table 1. Correlation, Means, and Standard Deviations of the Variables $(n=2286)$

\begin{tabular}{|c|c|c|c|c|c|c|c|c|c|}
\hline & 1 & 2 & 3 & 4 & 5 & 6 & 7 & Media & SD \\
\hline (in)Visibility & & & & & & & & 2.35 & .90 \\
\hline Worries of discrimination & $-.20^{* *}$ & & & & & & & 2.17 & 1.13 \\
\hline Discrimination as a problem & $-.25^{* *}$ & $.22^{* *}$ & & & & & 3.57 & .60 \\
\hline Treated with less respect than other people & -.14 & $-.27^{* *}$ & $-.13^{*}$ & $.50^{* * *}$ & & & $.11^{* *}$ & 2.68 & .63 \\
\hline People act as if you are not smart & $.10^{*}$ & $.31^{* *}$ & $.16^{* *}$ & $-.11^{*}$ & $-.14^{* *}$ & & .75 \\
\hline Blacks & $-.17^{* *}$ & $.40^{* *}$ & $.15^{* *}$ & $-.22^{* *}$ & $-.26^{* *}$ & $.44^{* *}$ & & .63 & .48 \\
\hline Male & .08 & $.14^{*}$ & -.01 & $-.10^{*}$ & $-.11^{* *}$ & .07 & $.21^{* *}$ & .65 & .48 \\
\hline
\end{tabular}


Discrimination is negatively associated with invisibility. The more discriminated black men perceived themselves to be, the less visible they perceived themselves to be. This result reflects Franklin's [2] assumptions.

Table 2. Standardized Factor Loadings for Variables

\begin{tabular}{|c|c|}
\hline & Lambda \\
\hline Perceived discrimination & \\
\hline Worries of discrimination & $1.0(.89)$ \\
\hline Discrimination as a problem & $.84(.65)$ \\
\hline Social Recognition & \\
\hline Treated with less respect & $.84(.72)$ \\
\hline People act as if you are not smart & $1.17(.63)$ \\
\hline
\end{tabular}

Numbers in parentheses represent standardized estimates. All estimates are significant at $\alpha=.001$

The measurement model is shown in Figure 2, which illustrates the results of the hypothesized relationship between variables, with standardized $\beta$ weight for all hypothesized paths. Results indicate a not significant maximum likelihood chi-square value, suggesting a good fit. However, the chi-square goodness of fit is not an accurate measure as it is sensitive to larger sample sizes. As the sample size increases, power also increases, and therefore the probability associated with the chi-square to detect significant, but meaningless differences fits between the hypothesized model and perfect model $[18,20]$. In contrast, the CFI and the RMSEA are more accurate as they are not sensitive to large sample size [20]. CFI exceeds .90, indicating an extremely good fit, and the RMSEA is equal to .05 , indicating a good fit $[18,19,20]$. According to these indexes, it is possible to conclude that the path model produces a very good fit.

Perceived discrimination. For black males, perceived discrimination has a negative and highly significant direct effect on visibility $(\beta=-.55, p<.01)$ and on social recognition $(\beta=-.65, p<.01)$. As perceived discrimination increases, the sense of visibility and social recognition decreases. There is also a highly significant and direct effect between discrimination and group affiliation $(\beta=.52, p$ $<.01$ ); as perceived discrimination increases, the sense of group affiliation increases. The relation between perceived discrimination and visibility is mediated by group affiliation, reducing the effect discrimination has on visibility $(\beta=-.1, p$ $<.05$ ). Increased levels of perceived discrimination lead to an increase in the sense of group affiliation, thus an increased sense of visibility $(\beta=.13, p<.05)$.

Social recognition. For black males, the more social recognition they perceived, the more the group affiliation ( $\beta$ $=.24, p<.01)$ and the higher the sense of social recognition, the higher the perception of visibility $(\beta=.13, p<.05)$. There is no significant relationship between social recognition and (in)visibility.

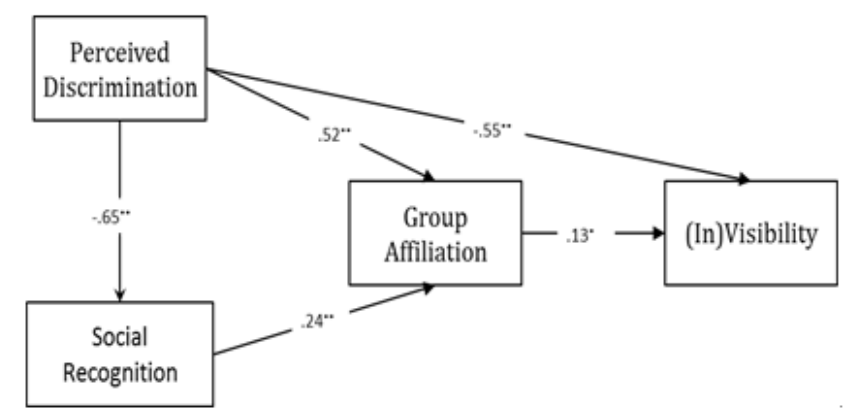

Figure 2. Path model testing direct and mediated relations among variables of interest. $\chi^{2}(18)=147.61, \mathrm{CFI}=0.97, \mathrm{NFI}=0.96$, NNFI $=0.91$, $\mathrm{CFI}=0.97, \mathrm{GFI}=0.99$, RMSEA $=0.05$. Values reflect standardized coefficients. ${ }^{* * *} p<0.001,{ }^{* *} p<0.01$, and $* p<0.05$.

The structural parameters reported in Table 3 represent the total and indirect effects of exogenous variables (gender and race) on latent constructs. In the present context, our interest is on black males and visibility.

Males have a significant effect on racial micro-aggression $(\beta=-.08, p<.05)$ and on (In)visibility $(\beta=-.12, p<.01)$. Men perceived less social recognition, yet are less visible than women. Black is significantly and positively related to perceived discrimination $(\beta=.59, p<.001)$ and group affiliation $(\beta=.44, p<.01)$.

Blacks perceive higher levels of discrimination and more sense of group affiliation than other races. Also, blacks perceive less social recognition $(\beta=-.32, p<.05)$ and are more invisible $(\beta=-.20, p<.05)$. Race is associated indirectly with social recognition, group affiliation, and visibility.

The results corroborate the hypothesis that race has a significant and indirect effect on visibility. Blacks perceived less visibility and less social recognition than other races.

Table 3. Standardized coefficient of Total and Indirect effect of exogenous variables with constructs

\begin{tabular}{|c|c|c|c|c|}
\hline \multirow{2}{*}{ Constructs } & \multicolumn{2}{|c|}{ Male } & \multicolumn{2}{c|}{ Black } \\
\cline { 2 - 5 } & $\begin{array}{c}\text { Total } \\
\text { effect }\end{array}$ & $\begin{array}{c}\text { Indirect } \\
\text { effect }\end{array}$ & $\begin{array}{c}\text { Total } \\
\text { effect }\end{array}$ & $\begin{array}{c}\text { Indirect } \\
\text { effect }\end{array}$ \\
\hline $\begin{array}{c}\text { Perceived } \\
\text { Discrimination }\end{array}$ & .05 & & $.59^{* * *}$ & \\
\hline Social recognition & $-.08^{*}$ & -.02 & $-.32^{* * *}$ & $-.42^{* *}$ \\
\hline Group affiliation & -.03 & -.01 & $.44^{* * *}$ & $.28^{* *}$ \\
\hline (In)Visibility & $-.12^{* *}$ & -.03 & $-.20^{* *}$ & $-.23^{* *}$ \\
\hline
\end{tabular}

$* * * p<0.001 * * p<0.01 * p<0.05$

\section{Discussion}

The proposed study is the first quantitative attempt to study Franklin's [2] invisible syndrome, on the importance of perceived discrimination, social recognition, and group affiliation to the perception of invisibility. Today, invisibility has become pervasive. Invisibility is a reality most racial/ethnic minorities experienced, with its subtle and increasingly customary form of expressions. Invisibility 
stands, based on the results of this study, for powerlessness and the disenfranchisement, which defines marginalized identities and behaviors that racial/ethnic minorities visible to the mainstream.

In these contexts, invisibility is impotence. What is powerful is to be seen.

Evidence of this study shows that perceived discrimination decreases social recognition. This result is in concordance with previous studies [2]. As mentioned before, social recognition depends on the vision of the dominant group within the social hierarchy. So, as black men perceived that they are invisible, due to discrimination and prejudice [2, $3]$, the sentiment of invisibility increases and with it the poor or none social recognition [2, 3]. Minorities also experienced hyper-visibility $[10,11]$. A clear example of this may be African American men who are hyper-visible to law enforcement. The degree to which racial/ethnic minorities experience invisibility or hyper-visibility would lead them to question the true impetus behind the social dynamic, as the vast majority struggle to maintain a white dominant order in U.S. society.

Based on these results, discrimination can be an important social stressor, leading to the development and escalation of both the sentiment of poor social recognition and invisibility among racial/ethnic minorities, which in turn can lead to enhanced social disruptions. It is not clear, however, that invisibility comes from exercise of power, as minorities are invisible not to obtain power but to escape from it. This form of micro-aggression modifies the sentiment a minority has towards society, affecting the manner minorities interact with others $[2,3]$ and perpetuate practices and beliefs that reproduce the racial power of white supremacy [1]. Future studies should address the ways in which racial power practices that perpetuate the racial power and normalizes whiteness operate at the individual level: how can invisibility function as both a strategy of empowerment and/or a result of powerlessness?

Cultural differences can result in poor social recognition between the minority and majority populations. Results show that the higher perceptions of racial discrimination are positively related to poor social recognition, or racial micro-aggression. Lack of social recognition is a covert from of racism, which entails a pejorative message, whether verbal or nonverbal, intentional or unintentional, that the other person's cultural or ethnic/racial heritage is not being recognized [8]. Although, this form of micro-aggression is most similar to the concept of aversive racism in that the perpetrator means no harm and thus is unaware of the demeaning exchange $[8,21]$, racial/ethnic minorities perceive as evident the lack of social recognition, which in turn makes them invisible [2, 12]. The resultant racial micro-aggression, as Smith and colleagues [14] manifested, produces emotional, psychological, and physiological distress, or racial battle fatigue. The racial battle fatigue framework, explains Smith and his colleagues [14], demonstrates that racial/ethnic minorities experience mundane racial micro-aggression that are built up over time and are detrimental to their overall quality of life.

The results of this study support Franklin's [2] assumptions. Evidence documented the relation between perceived discrimination and group affiliation. Group affiliation is a response to discrimination and a strategy to dilute the distress caused by discrimination. Additionally, in these racial/ethnic groups, the sense of belonging is developed and the experiences which are a product of discrimination and poor social recognition are invalidated. Through sanity check, as Franklin et al 2000 named the process that allows racial/ethnic minorities to cope with discrimination, blacks develop a social awareness of discriminatory behavior and develop mechanisms to overcome the feeling of (in)visibility [5], protecting their mental well- being [3]. In fact, the results of this study show that group affiliation is a mediator for the influences of perceived discrimination and poor social recognition on the sentiment of invisibility. Visible minority status, on the other hand, may also enhance alienation and in some cases lead to actual social isolation $[5,9]$. So, to reduce racism outcomes, such as aggressive behaviors, it is necessary to reinforce black people's sense of social recognition and group affiliation, providing them with a sense of respect and dignity. At the same time, it is important not to fall into the over recognition of blacks, as this hypervisibility is a covert form of racism. Although group affiliation is one way minorities can advance, people need to achieve intellectual liberation, which comes through rigorous and scientific based understanding of the social dynamic.

Although racism's association with the invisibility syndrome is not as clear cut as institutional racism, it is just as painful because social and cultural divisions still exist in our society, where whites consciously reject racial prejudice and discrimination, while unconsciously responding according to stereotypical beliefs. The importance of the perception of discrimination with respect to visibility is demonstrated in this study. Therefore, it is necessary to develop positive racial encounters based on the exaltation of a racial/ethnic minority culture instead of ignoring its racial attributes.

In conclusion, the United States still has a long way to go to combat racism and discrimination. Not with policies, but with education, with a scientific knowledge and understanding of the social dynamic that perpetuates racism and the racial power. It is important therefore to encourage intellectual understanding of the mechanism through which individual behavior perpetuates racism and the strategies minorities used to overcome discriminatory experiences. Similarly, future studies should focuses on the group affiliation and the apparatus through which group affiliation modifies minorities' experiences of (in)visibility. However, the lack of a databases from which qualitative studies can develop from, make it difficult for scholars to address these issues. Policies should therefore incentive the creation of adequate databases from which to understand the impact of 
discrimination at the micro-level.

Policies should also encourage the formation of, and affiliation to, racial/ethnic support groups. The racial support groups will promote and open, neutral, and safe space where conversation about discriminatory experiences, racism and privilege are vent. These groups will encourage the dialogue within racial/ethnic minorities and, according to Yeh [21], racial/ethnic minorities' sense of respect and dignity, as well as their racial/ethnic identity are reinforced. These groups should come to the fore of social changes and radical knowledge about racial/ethnic dynamic, which is fundamental as it is a buffer against racism $[2,3]$ and the once negative experience of discrimination will be utilized to help determine a new perspective on cross-racial interpersonal encounters, explains Franklin [2]. The conclusion of the dialogues should be translated into programs of racial/ethnic awareness and diversity, improving consequently the psychological well- being of minorities [14].

One of the fundamental products of the Civil Rights' movement is the enactment of anti-discrimination legislations. These legislations seeks to protect the right of racial/ethnic minorities; however, most minorities experience a "separate but equal life" as they are either ignored or kept distant, perpetuating the negative effects of the era Jim Crow era [1]. Todays' society is characterized by a subtle and seemingly non-racial behavior adopted by whites. It is necessary, therefore, to recognize that racism has evolved from an overt to a more hidden form, disguised in the discourse of "I do not see color" to a more deleterious expression of "I do not see people of color." This new form of racism, where minorities are completely invisible, is a social response to the multiple policies that attack discrimination openly. By informing how racism is more than an obvious and evident deliberate behavior, it is expected that these racial/ethnic support groups will highlight the importance of recognizing the other, not for their differences, but for their commonalities, consequently reducing social stressors caused by discrimination and the lack of social recognition. In fact, the results of this study show that group affiliation is a mediator of the influences of perceived discrimination and poor social recognition on the sentiment of invisibility.

Consequently, invisibility must be socially recognized. Future studies should focus on the relevance of visibility, and the multiple variants; invisibility, hypervisibility, or hypervisibility, as an outcome of discrimination and the implication of it on racial/ethnic groups. This study is only a starting point for examining the potential added value of group affiliation as a dynamic for understanding the social dynamics of visibility. Theoretically, the findings support the visible paradox that African Americans face every day proposed by Franklin [2].

With regard to the study's limitations, unfortunately the data set was not designed for measuring visibility. However the author was able to investigate this phenomenon.
Furthermore, scholars are encouraged to propose quantitative studies that gather data on racial micro-aggression, perceived discrimination, and group affiliation and how these produce racial visibility or invisibility.

\section{Acknowledgements}

I would like to thank Professor Gerald Schwartz for their helpful comments on an earlier draft of this paper.

\section{REFERENCES}

[1] E. Bonilla-Silva. Racism without Racists: Color-Blind Racism and the Persistence of Racial Inequality in the United States. Lanham, MD: Rowman \& Littlefield Publishers, United States, 2006.

[2] A.J. Franklin. Invisibility Syndrome and Racial Identity Development in Psychotherapy and Counseling African American Men, The Counseling Psychologist, Vol. 27, No. 6, 761-793, 1999.

[3] A.J. Franklin, N. Boyd-Franklin. Invisibility Syndrome: A Clinical Model of the Effects of Racism on African-American Males, American Journal of Psychiatry, Vol. 70, No. 1, 33-41, 2000 .

[4] T.A. Parham. Invisibility Syndrome in African Descent People: Understanding the Cultural Manifestations of the Struggle for Self-Affirmation, The Counseling Psychologist, Vol. 27, No. 6, 794-801, 1999.

[5] K.B. McDonald, A.M.H. Wingfield. (In)visibility Blues: The Paradox of Institutional Racism, Sociological Spectrum, Vol. 50, 28-50, 2009.

[6] W. E. B. Du Bois. Dusk of Dawn: An Essay toward an Autobiography of Race Concept, Schocken Books, New York, 1968.

[7] R. Ellison. Invisible Man, Random House, New York, 1952.

[8] D. W Sue, C.M. Capodilupo, G.C. Torino, J.M. Bucceri, A.M. Holder, K.L. Nadal, M. Esquilin. Racial Microaggression in Everyday Life: Implications for Clinical Practice, American Psychologist, Vol. 62, No. 4, 271-286, 2007.

[9] C. W. Mills. Blackness Visible: Essays on Philosophy and Race, Cornell University Press, Ithaca, NY, 1998.

[10] G.E. Wyatt. Beyond Invisibility of African American Males: The Effects on Women and Families, The Counseling Psychologist, Vol. 27, No. 6, 803-810, 1999.

[11] F. Tuitt. Enhancing Visibility in Graduate Education: Black Women's Perceptions of Inclusive Pedagogical Practices, International Journal of Teaching and Learning in Higher Education, Vol. 22, No. 3, 246-257, 2010.

[12] W. Sun, W. J. Starosta. Perceptions of Minority Invisibility among Asian American Professionals, The Howard Journal of Communications, Vol. 17, No. 2, 119-142, 2006 
[13] B. Orfali. Active Minorities and Social Representations: Two Theories, One Epistemology, Journal for the Theory of Social Behaviour, Vol. 32, No. 4, 395-416, 2002.

[14] W.A. Smith, M. Hung, J.D. Franklin. Racial Battle Fatigue and the Miseducation of Black Men: Racial Microaggression, Societal Problems, and Environmental Stress, The Journal of Negro Education, Vol. 80, No. 1, 63-82, 2011.

[15] African American Men Survey. Washington, DC: The Henry J. Kaiser Family Foundation, 2006.

[16] B.M. Byrne. Structural Equation Modeling with LISREL, PRELIS, and SIMPLIS: Basic Concepts, Applications, and Programming, Lawrence Erlbaum, Mahwah, New Jersey, 1998.

[17] H. B. Kaplan, R. J. Johnson. Social Deviance: Testing a General Theory, Kluemer Academic/Plenum Publishers, New
York, 2001

[18] L. Hu, P.M. Bentler. Cutoff Criteria for Fit Indexes in Covariance Structure Analysis: Conventional Criteria versus New Alternatives, Structural Equation Modeling, Vol. 6, No. $1,1-55,1999$.

[19] X. Fan, B. Thompson, L. Wang. Effects of Sample Size, Estimation Methods, and Model Specification on Structural Equation Modeling Fit Indexes, Structural Equation Modeling, Vol. 6, No. 1, 56-83, 1999.

[20] R.C. MacCallum, M. W. Browne, H. M. Sugawara. Power Analysis and Determination of Sample Size for Covariance Structure Modeling, Psychological Methods, Vol. 1, No. 2, 130-149, 1996.

[21] C.J. Yeh. Invisibility and Self-Construal in African American Men: Implications for Training and Practice, Counseling Psychologist, Vol. 27, No. 6, 810-819, 1999. 